\title{
La política de dividendos y la rentabilidad de los negocios
}

\section{RESUMEN}

Las empresas, desde el punto de vista financiero, para maximizar el valor de las acciones representativas de su capital social, buscan compatibilizar sus decisiones financieras en cuanto a inversión financiamiento y dividendos. La política de dividendos, sea en efectivo y/o en acciones liberadas, es determinante en la percepción del valor de mercado de la empresa.

La evidencia práctica del efecto de la política de dividendos en el valor de las acciones de la empresa, se presenta para una muestra de cuatro empresas de generación eléctrica del país: ELECTROPERU, EDEGEL, ENERSUR y EGENOR. La producción de energía por estas empresas en el año 2006, según el Comité de Operación Económica del Sector Eléctrico (COES), representó el $75 \%$ de la producción del Sistema Eléctrico Interconectado Nacional. Las cuatro empresas cuentan con política de dividendos que guardan cierta similitud, y cuya aplicación en cada ejercicio fiscal depende de los planes de crecimiento y/o de optimización de pasivos que apliquen.

Palabras clave: Dividendos, valor, rentabilidad, costo de oportunidad, acciones, capital social, patrimonio.

DIVIDENDS POLICY AND PROFITABILITY IN BUSINESSES

\section{ABSTRACT}

The companies, of the financial point of view, to increase to the maximum the value of the representative actions of their social capital, look for to coordinate their financial decisions as for the investment, financing and dividends. The politics of dividends, be cash the money and/or in the additional actions about utilities, it is strong in the perception of the value of market of the company. The practical evidence of the politics's of dividends effect in the value of the actions of the company, is presented, for a sample of four companies of generation electric maidservant: ELECTROPERU, EDEGEL, ENERSUR AND EGENOR. The energy production for these companies in the year 2006 according to the Committee of Economic Operation of the Electric Sector (COES) it represented $75 \%$ of the production of the National Cruz-trigger the Electric System. The four companies have the politics of dividends that the you/they keeps certain similarity, and of who the application in each fiscal exercise depends on the plans of growth and/or o optimization of passive that apply.

Keywords: The Dividends, the value, the profitability, the opportunity cost, the actions, the social capital, the patrimony.

\section{INTRODUCCIÓN}

Una persona con capital o inversionista que cuenta con excedente de dinero, busca rentabilizarlo invirtiendo en alternativas de negocios que le reporten un rendimiento o retorno adecuado a sus expectativas, asumiendo un nivel razonable de riesgo. La metodología de análisis usual de las alternativas de inversión consiste en proyectar el flujo de caja del negocio que le correspondería al inversionista -bajo determinadas premisas- y analizar este flujo con una tasa de descuento que represente el rendimiento mínimo requerido o deseado por el inversionista para aceptar la inversión analizada.

Si el negocio a desarrollar corresponde a una sola persona o esta persona tiene el control mayoritario de las acciones de la empresa, la metodología de análisis de inversiones señalado anteriormente, responde adecuadamente. El tema a discutir es cuando el inversionista tiene participación minoritaria en la empresa, en donde el rendimiento de su aporte de capital dependerá de la política de distribución de dividendos que aplique la empresa.

En el presente artículo, analizaremos las implicancias para el inversionista, llamado también accionista o socio, de la política de dividendos de la empresa en la rentabilidad esperada de la alternativa de inversión que elija. Es de tener en cuenta que la política de dividendos se aplica a la utilidad neta de cada ejercicio fiscal y su pago en efectivo a favor de los accionistas representa un egreso que afecta la disponibilidad de caja de la empresa, con lo cual dicha empresa dispondrá de menos recursos financieros para invertir o para afrontar sus obligaciones con terceros (principalmente por endeudamiento en la ejecución de inversiones).

Toda empresa requiere mostrar con claridad su política de dividendos para atraer inversionistas mediante el aporte de capital fresco, condición que es imperativa en empresas cuyas acciones listan o cotizan en la Bolsa de Valores de Lima (BVL). En tal sentido, como aplicativo, analizaremos la política de dividendos de las principales empresas de generación eléctrica registradas en dicha Bolsa.

Naturalmente, para que una persona decida invertir en un negocio intervienen una serie de consideraciones, una de ellas es la rentabilidad. En tal sentido, validaremos nuestra investigación en función al efecto de la política de dividendos en la rentabilidad esperada por el inversionista en la alternativa de inversión analizada.

Magister en Administración. Profesor en la Facultad de Ingeniería Industrial, Departamento Académico de Producción y Gestión Industrial de la UNMSM. E-mail: nestor_sj@hotmail.com 


\section{LA POLÍTICA DE DIVIDENDOS}

Por el aporte de capital de los inversionistas, que constituye el capital social de la empresa, dicha empresa emite acciones según la proporción del aporte. El testimonio del capital social, con indicación del número de acciones y/o el valor nominal de cada acción, conjuntamente con el estatuto social de la empresa se eleva a escritura pública y se registran en los Registros Públicos. Los tenedores de estas acciones se constituyen en accionistas, quienes encargan la gestión de la sociedad empresarial al directorio, el cual, a su vez, designa al gerente general, quien es el representante legal de la empresa. Los accionistas se reúnen por lo menos una vez al año, para evaluar la gestión de la sociedad (empresa), aprobar los estados financieros del periodo fiscal anterior y adoptar otras decisiones de interés para la sociedad.

La política de dividendos corresponde ser aprobada por la Junta General de Accionistas de la empresa, en concordancia con su estatuto social y la Ley General de Sociedades. Para la aplicación de esta política a la utilidad neta del ejercicio fiscal, determinado en el estado de ganancias y pérdidas, se deduce el $10 \%$ por concepto de reserva legal, denominándose al saldo resultante, utilidad distribuible. La obligación de deducir un porcentaje de la utilidad anual para constituir la reserva legal, existe hasta que la reserva legal acumulada de la empresa sea equivalente al $20 \%$ de su capital social.

De esta manera, la política de dividendos se puede traducir en la distribución de dividendos por el 100\% de la utilidad distribuible o de una porción de ella. La parte de la utilidad distribuible que no se entregue en efectivo a los accionistas pasará a formar parte del patrimonio de la empresa como resultados acumulados (utilidades y/o pérdidas de periodos anteriores y del ejercicio corriente). Posteriormente, los resultados acumulados se pueden distribuir como dividendos en efectivo o pueden ser capitalizados (incorporados al capital social) o puede optarse por una combinación de ambos conceptos (pago de dividendos en efectivo y en acciones). El pago de dividendos en efectivo es un egreso de caja para la empresa, dando lugar a la reducción del patrimonio y del activo por el mismo monto (dividendos pagados).

Si los resultados acumulados se capitalizan, el patrimonio no varía y tampoco existe salida de efectivo (egreso de caja), lo que permite fortalecer patrimonialmente a la empresa; toda vez que con el recurso equivalente de la capitalización (dividendos no entregados en efectivo), la empresa puede adquirir ac- tivos y/o prepagar obligaciones financieras, según las condiciones de los mercados financiero y de capitales. En esta situación, el sacrificio del accionista, de dejar de percibir dividendos en el presente, debería ser compensado ampliamente por las ganancias futuras que le ofrezca la empresa.

La capitalización de utilidades puede ser mediante la emisión de acciones, lo cual implica mantener el precio nominal de las acciones, o contemplarse el cambio del valor nominal, en donde el número de acciones se mantiene. Si el accionista recibe dividendos en acciones tiene la opción de convertirlo en efectivo, al entregarlo en venta directa a otro inversionista o mediante su venta en la BVL si las acciones cotizan.

Sobre el particular, comentemos el siguiente caso como aplicativo. Una empresa al cierre de un ejercicio fiscal tiene un activo por S/. 100000 con una relación deuda a capital de 1 a 4, donde su capital social es S/. 40000 (constituido por 40000 acciones a un nuevo sol por acción de valor nominal) y la reserva legal asciende a S/.5000. En estas condiciones, la empresa acuerda distribuir dividendos en efectivo, por el equivalente al $75 \%$ de la cuenta resultados acumulados del patrimonio, capitalizar el otro $15 \%$ y el saldo mantenerlo en dicha cuenta:

a. El activo de la empresa (S/. 100000$)$ está financiado con S/. 20000 de pasivo y S/. 80000 de patrimonio, donde esta última cuenta está conformada por S/. 40000 de capital social, S/. 5000 de reserva legal y S/. 35000 de resultados acumulados.

b. Con la estructura indicada del patrimonio, cada acción tiene un valor nominal de S/. 1 y un valor contable (patrimonio / número de acciones) de S/. 2 por acción.

c. Al distribuirse dividendos en efectivo por S/. 26 250 ( $75 \%$ de S/. 35 000), el patrimonio se reduce a S/. 53750 , el nuevo valor del activo disminuye a S/. 73750 (por egreso de caja debido al pago de dividendos) y el pasivo se mantiene en S/. 20 000 .

d. Hasta este momento, el nuevo valor de resultados acumulados es S/. 8750 (deducida la entrega a accionistas de dividendos en efectivo). Luego, con la capitalización de S/. 5250 (equivalente al $15 \%$ de los resultados acumulados iniciales), el capital social se eleva a S/. 45 250, y el saldo final de resultados acumulados queda en S/. 3500. 


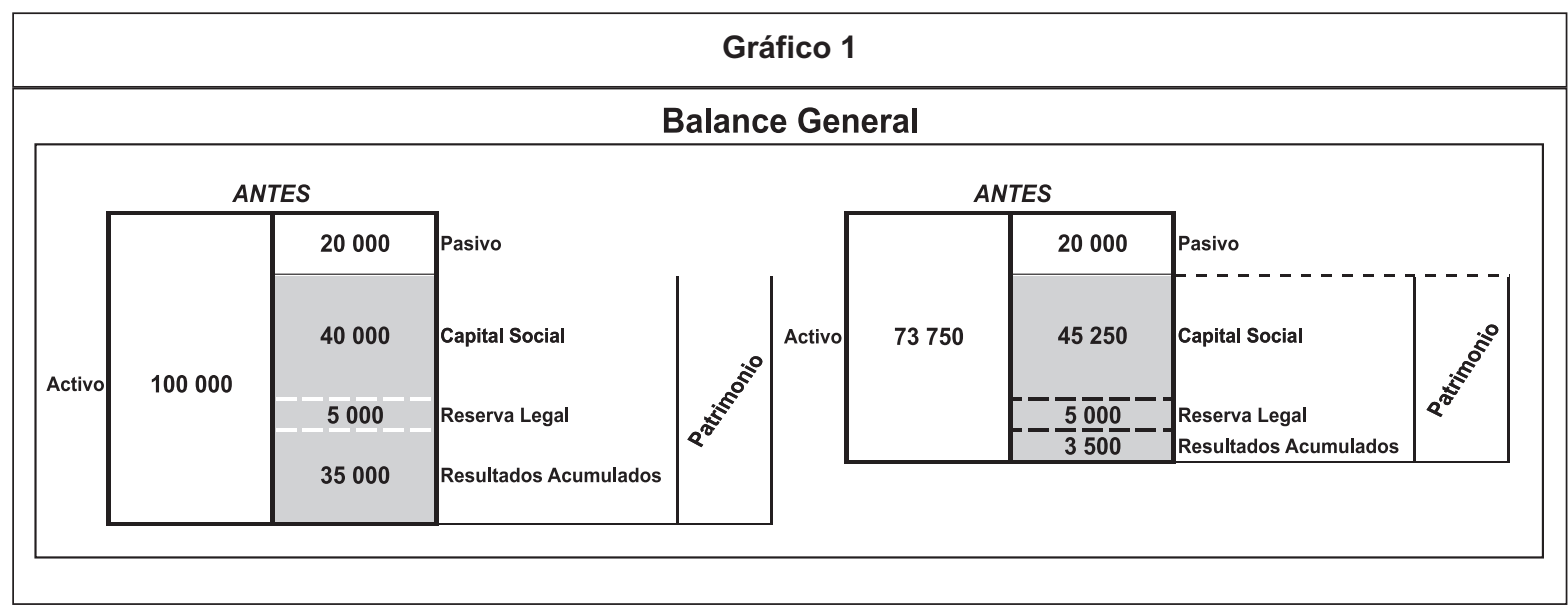

Fuente: Elaboración propia

e. De esta manera, la estructura del nuevo patrimonio de la empresa por S/. 53750 (luego de la distribución de dividendos en efectivo y la capitalización de utilidades) está integrada por S/. 45250 de capital social, S/. 5000 de reserva legal y S/. 3500 de resultados acumulados.

f. En el Gráfico N.$^{\circ} 1$ se muestra la estructura comparativa del balance general de la empresa, antes y después de la aplicación de la política de dividendos, consistente en la distribución de dividendos en efectivo y capitalización de una parte de las utilidades.

g. En cuanto a los efectos de la capitalización de utilidades, se tiene lo siguiente:

- Si la empresa mantiene el valor nominal en S/. 1 la acción, se tendrán 45250 acciones (40 000 las acciones iniciales y 5250 acciones liberadas por la capitalización). En este caso, la empresa libera a favor de sus accionistas, 5250 acciones a un valor nominal de S/. 1, y de esta manera el valor contable de las acciones se reduce a S/. 1,18785 por acción.

- En cambio, si por la referida capitalización, se mantiene el número inicial de 40000 acciones, se tiene que modificar el valor nominal a S/. 1,13125 por acción. Bajo este escenario, los accionistas no reciben acciones por la capitalización de utilidades, solo que el valor contable de cada acción es de S/. 1,34375.

Del caso comentado, deducimos que una vez que la empresa ha obtenido utilidades (resultados económicos netos positivos), la aplicación de la política de dividendos afecta la posición patrimonial de la empresa, dependiendo si el pago de dividendos es en efectivo o en acciones, lo cual, a su vez, influye en el mercado de valores si las acciones de la empresa cotizan (se transan - compran y venden) en la BVL.

\section{LOS EFECTOS DE LA POLÍTICA DE DIVIDEN- DOS EN LA RENTABILIDAD}

Los inversionistas, para decidir invertir en una alternativa de inversión y/o comprar acciones en la BVL, no solo analizan los estados financieros de la empresa, sino que requieren conocer las expectativas de generación de ganancias futuras de la empresa, es decir su valor de mercado.

Una manera de estimar el valor de mercado de las acciones consiste en hallar el valor presente neto (VPN) del flujo de caja esperado por el inversionista tenedor de las acciones, considerando el monto invertido y el valor de recupero del mismo, luego de un horizonte finito de proyección. También se puede utilizar como criterio de análisis del valor la tasa interna de retorno $(T I R)$ de la inversión.

Para tal efecto, asumamos una alternativa de inversión que demanda una inversión en activos fijos por S/. 90000 (que se deprecia al 10\% anual) y de capital de trabajo por S/. 10 000, donde el valor residual de estas inversiones, luego de la operación comercial del negocio durante cinco años, equivale a su valor en libros contables al cierre del año 5. Los ingresos operativos netos desembolsables (antes de la depreciación) se proyectan en S/. 18000 en el primer año, S/. 24000 el segundo año, y S/. 32000 anuales en los siguientes tres años. El $60 \%$ de la inversión total se financia con una deuda bancaria a la tasa de interés de $10 \%$ anual, a ser amortizada en tres cuotas de capital constante al final de cada año luego de transcurridos dos años de gracia (periodo donde se paga solo intereses). 


\begin{tabular}{|c|c|c|c|c|c|c|c|}
\hline \multicolumn{8}{|c|}{ Cuadro 1.} \\
\hline \multicolumn{8}{|c|}{ Estado de Ganancias y Pérdidas } \\
\hline Concepto & 0 & 1 & 2 & 31 & 4 & 5 & 6 \\
\hline Ingresos operativos netos & & 18000 & 24000 & 32000 & 32000 & 32000 & \\
\hline Depreciación & & 9000 & 9000 & 9000 & 9000 & 9000 & \\
\hline Intereses deuda & & 6000 & 6000 & 6000 & 4000 & 2000 & \\
\hline Utilidad antes impuesto a la renta & & 3000 & 9000 & 17000 & 19000 & 21000 & \\
\hline Impuesto a la renta & & 900 & 2700 & 5100 & 5700 & 6300 & \\
\hline Utilidad neta & & 2100 & 6300 & 11900 & 13300 & 14700 & \\
\hline
\end{tabular}

Asimismo, la empresa que implemente esta alternativa de inversión estará afecta al pago del impuesto a la renta, y tiene como política de dividendos entregar dividendos en efectivo por el $80 \%$ de la utilidad distribuible del ejercicio (luego de deducir $10 \%$ de reserva legal); el pago de los dividendos generados en un ejercicio fiscal se concreta en el ejercicio inmediato siguiente. El costo de oportunidad de los accionistas se estima en $12 \%$ anual (retorno mínimo esperado).

Con estas premisas para estimar la rentabilidad de la alternativa de inversión para sus accionistas, se tiene lo siguiente:

\section{a. Rentabilidad financiera de la alternativa de in- versión}

En el Cuadro N..$^{\circ} 1$ se determina la utilidad neta anual que generaría la alternativa analizada, la cual viene a ser el resultado económico esperado del negocio durante un horizonte de operación comercial de cinco años.

La proyección de la generación neta de efectivo (caja), incluyendo el efecto de la deuda y sin contemplar la política de dividendos, se muestra en el Cuadro N. ${ }^{\circ} 2$. A esta presentación la denominaremos modelo base, y su finalidad es solo para apreciar, después, el impacto que tendría dicha política en la rentabilidad de la inversión para sus accionistas.
Los resultados financieros mostrados en el Cuadro $\mathrm{N} .^{\circ} 2$ constituyen el flujo de fondos de la empresa, el cual es una reconstrucción aproximada -a partir de la utilidad neta- del saldo neto de caja que se obtiene según el flujo de caja, donde se asume que no existen inventarios y que todas las operaciones comerciales son al contado. En cuanto al año en que se incorpora el valor residual al flujo de caja, se asume que el negocio opera comercialmente hasta el cierre del año 5, por lo que la "liquidación" de los activos y pasivos de la empresa se materializa durante el año 6. El monto del valor residual se asume por el equivalente al valor en libros de los activos fijos al cierre del año 5, y neto de los gastos operativos necesarios para su efectivización en el sexto año.

Asimismo, se contempla como supuesto que los accionistas tengan el control absoluto del negocio, por lo que adoptarán las mejores decisiones en cuanto al destino del saldo neto de caja que resulte de cada periodo proyectado. Bajo el supuesto que el saldo neto de caja de cada periodo sea de libre disposición para los accionistas de la empresa, la rentabilidad (TIR) de la alternativa de inversión resulta atractiva, toda vez que supera al valor del costo de oportunidad de los accionistas ( $12 \%$ anual).

TIR del Modelo Base: $21,1 \%$ anual

\begin{tabular}{|c|c|c|c|c|c|c|c|}
\hline \multicolumn{8}{|c|}{ Cuadro 2.} \\
\hline \multicolumn{8}{|c|}{ Flujo de caja } \\
\hline Concepto & 0 & 1 & 2 & 3 & 4 & 5 & 6 \\
\hline Utilidad neta & & 2100 & 6300 & 11900 & 13300 & 14700 & \\
\hline (+) Depreciación & & 9000 & 9000 & 9000 & 9000 & 9000 & \\
\hline$(+)$ Deuda & 60000 & & & & & & \\
\hline$(+)$ Valor residiual & & & & & & & 55000 \\
\hline (-) Inversión total & 100000 & & & & & & \\
\hline (-) Amortización de capital deuda & & 0 & 0 & 20000 & 20000 & 20000 & \\
\hline Saldo neto de caja & -40000 & 11100 & 15300 & 900 & 2300 & 3700 & 55000 \\
\hline
\end{tabular}




\begin{tabular}{|c|c|c|c|c|c|c|c|}
\hline \multicolumn{8}{|c|}{ Cuadro 3.} \\
\hline \multicolumn{8}{|c|}{ Distribución de la Utilidad Neta } \\
\hline Concepto & 0 & 1 & 2 & $3 \mid$ & 4 & 5 & 6 \\
\hline Utilidad neta & & 2100 & 6300 & 11900 & 13300 & 14700 & 0 \\
\hline Reserva legal & & 210 & 630 & 1190 & 1330 & 1470 & \\
\hline Utilidad distribuible & & 1890 & 5670 & 10710 & 11970 & 13230 & \\
\hline Dividendos & & 1512 & 4536 & 8568 & 9576 & 10584 & \\
\hline Saldo a resultados acumulados & & 378 & 1134 & 2142 & 2394 & 2646 & \\
\hline
\end{tabular}

\section{b. Rentabilidad de la alternativa de inversión para los accionistas}

Ahora, incorporemos en nuestro análisis el efecto de la política de dividendos, para lo cual construimos el flujo de caja para los accionistas y el flujo de caja para la empresa luego del pago de dividendos. Cuando la empresa paga dividendos en efectivo debe retener a los accionistas el impuesto de 4,1\% del monto pagado como dividendos, la excepción es cuando el titular de dichas acciones es una persona jurídica domiciliada en el país. A su vez, la empresa, según el saldo final de caja de cada periodo (igual al saldo de la caja al final del periodo anterior más saldo neto del periodo), deberá endeudarse a corto plazo para cubrir el déficit que se presente por el pago de dividendos y/o buscará rentabilizar los excedentes de efectivo según los productos financieros que ofrezca el mercado y que sean concordantes con el perfil de riesgo de la empresa; los costos y rendimientos de estas operaciones, en la alternativa de inversión analizada, no se contemplan.
- En el Cuadro N. ${ }^{\circ} 3$, se calcula la distribución de la utilidad neta considerando la política de dividendos. Así a la utilidad neta se deduce el 10\% como reserva legal y del saldo (utilidad distribuible), el $80 \%$ corresponde a dividendos por pagar en efectivo.

- Teniendo en cuenta que el pago de dividendos se realiza una vez cerrado el ejercicio fiscal, pudiendo contemplarse pagos anticipados (pagos a cuenta), en el Cuadro N. ${ }^{\circ} 4$, se tiene el flujo de caja de la empresa luego del pago de dividendos. Para tal efecto, al saldo neto de caja del Modelo Base (el utilizado para estimar la rentabilidad financiera de la inversión) se deduce el pago realizado por concepto de dividendos (que comprende el neto pagado a los accionistas y la retención de impuestos, la cual después la empresa la transfiere a la SUNAT).

- Ahora, en el Cuadro N. ${ }^{\circ}$, se tiene el flujo de caja para los accionistas, en donde el costo para el accionista es el aporte de capital que realiza en la empresa al inicio para financiar las inversiones y el beneficio está representado por los

\begin{tabular}{|c|c|c|c|c|c|c|c|}
\hline \multicolumn{8}{|c|}{ Cuadro 4.} \\
\hline \multicolumn{8}{|c|}{ Flujo de caja de la empresa luego del pago de dividendos } \\
\hline Concepto & 0 & 1 & 2 & 3 & 4 & 5 & 6 \\
\hline Saldo neto de caja del Modelo Base & & 11100 & 15300 & 900 & 2300 & 3700 & 55000 \\
\hline (-) Pago dividendos en efectivo & & 0 & 1512 & 4536 & 8568 & 9576 & 10584 \\
\hline & & 11100 & 13788 & -3636 & -6268 & -5876 & 44416 \\
\hline Caja inicial & & 0 & 11100 & 24888 & 21252 & 14984 & 9108 \\
\hline Caja final & & 11100 & 24888 & 21252 & 14984 & 9108 & 53524 \\
\hline
\end{tabular}

\begin{tabular}{|c|c|c|c|c|c|c|c|}
\hline \multicolumn{8}{|c|}{ Cuadro 5.} \\
\hline \multicolumn{8}{|c|}{ Flujo de caja para los accionistas } \\
\hline Concepto & 0 & 1 & 2 & 3 & 4 & 5 & 6 \\
\hline Aporte de capital & -40000 & & & & & & \\
\hline Dividendos percibidos (a) & & 0 & 1450 & 4350 & 8217 & 9183 & 10150 \\
\hline Valor residual de la empresa (b) & & & & & & & 53524 \\
\hline Flujo neto para el accionista & -40000 & 0 & 1450 & 4350 & 8217 & 9183 & 63674 \\
\hline \multicolumn{8}{|c|}{$\begin{array}{l}\text { (a) Dividendos pagados por la empresa neto del impuesto a dividendos. } \\
\text { (b) Caja final de la empresa al final del horizonte de proyección. }\end{array}$} \\
\hline Fuente: Elaboración propia. & & & & & & & \\
\hline
\end{tabular}


dividendos (neto de impuesto) percibidos anualmente y por el valor residual de la empresa (saldo de caja acumulada al final del horizonte de proyección, es decir, disponibilidad de efectivo que no fue entregada a los accionistas, la cual es de propiedad de los accionistas).

De esta manera, se obtiene que la rentabilidad de la empresa para los accionistas, según la política de dividendos aplicada.

\section{TIR Accionistas: $15,4 \%$ anual}

Es de observar que en el flujo de caja para los accionistas, se considera en el año 6 , el valor residual del activo en efectivo que obra en poder de la empresa al cierre del año 5 (caja final acumulada a su valor nominal, sin contemplar rendimientos de dichos saldos durante los años proyectados).

De esta manera, conociendo el impacto de la política de dividendos, es posible aumentar la rentabilidad para los accionistas (TIR Accionistas: 15,4\% anual), mediante decisiones empresariales que permitan aproximarse a la rentabilidad potencial del negocio (TIR del Modelo Base: 21,1\% anual). Entre las principales decisiones destacan las siguientes:

- Obtener con los saldos de caja (Caja final) de cada periodo, rendimientos que satisfagan las expectativas de retorno de la empresa.

- Promover planes de inversión para el crecimiento de la empresa y/o plan de prepago de la deuda, acorde con las expectativas de rendimiento de los accionistas por su capital.

- Propiciar la entrega adicional de dividendos a los accionistas, vía la reducción del capital de la em- presa, siempre que las dos opciones anteriores resulten limitadas o poco atractivas.

\section{c. Comparación entre la rentabilidad financiera y la rentabilidad para los accionistas}

La diferencia entre la rentabilidad financiera de la inversión (estimada con la TIR del Modelo Base) y la rentabilidad efectiva para los accionistas (TIR Accionistas), obedece a que la TIR financiera toma como base la generación neta de efectivo de la inversión sin tener en cuenta la oportunidad y la forma de pago de dividendos; en cambio, la TIR para los accionistas considera el ingreso neto que reciben los accionistas según la política de dividendos que aplique la empresa y considera los impuestos a que está afectos el pago de dividendos en efectivo.

\section{TIR del Modelo Base: $21,1 \%$ anual TIR Accionistas: $15,4 \%$ anual}

En el Gráfico N.$^{\circ} 2$ se aprecia la evolución de la rentabilidad financiera de la alternativa de inversión (TIR del Modelo Base) y la rentabilidad para el accionista con aplicación de la política de dividendos (TIR Accionistas).

\section{LA POLÍTICA DE DIVIDENDOS DE EMPRESAS LISTADAS EN LA BOLSA}

La política de dividendos, especialmente la de distribuir ganancias en efectivo, no es uniforme para las empresas y puede variar con el tiempo, depende de la situación económica (utilidades) y financiera (disponibilidad de efectivo) de la empresa. La estructura accionaria de la empresa, que es determinante en la toma de decisiones, también influye en la aprobación y aplicación de esta política.

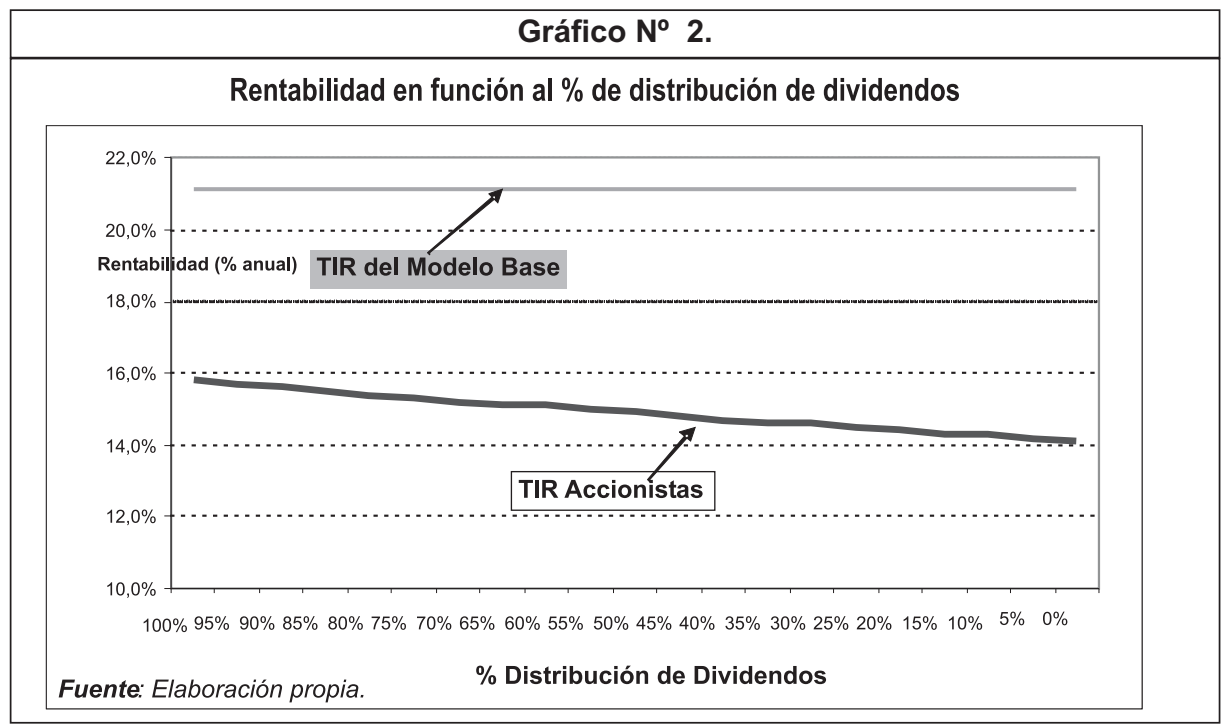


Para apreciar la importancia de la política de dividendos en la gestión de las empresas y en la creación de valor para sus accionistas, analizaremos la situación de cuatro empresas de generación eléctrica del mercado nacional, cuyas acciones están listadas en la BVL, ellas son: ELECTROPERU, EDEGEL, ENERSUR y EGENOR; la primera es una empresa estatal y las siguientes tres empresas son privadas y sus accionistas mayormente son inversionistas extranjeros.

\section{a. Producción, capacidad instalada y propiedad de las empresas}

De acuerdo al anuario estadístico 2006, publicado por el Organismo Supervisor de la Inversión en Energía y Minería (OSINERGMIN), en el año 2006, en el sector eléctrico se contaba con catorce (14) empresas de generación eléctrica, quienes produjeron 24686 GWh de energía, de los cuales, el $74,7 \%$ correspondió a las cuatro empresas de la muestra seleccionada; y los otros $25,3 \%$ fueron explicados por diez empresas (cinco estatales y cinco privadas), según se aprecia en el Gráfico N. ${ }^{\circ} 3$.

ELECTROPERU es una empresa estatal (el $100 \%$ de sus acciones pertenecen al Fondo Consolidado de Reservas previsionales - FCR); básicamente, su producción es hidroeléctrica. El principal accionista de EDEGEL es Generandes Perú S.A. (55,44\%), quien forma parte del grupo Endesa de España. El accionista mayoritario de ENERSUR es el grupo Suez de Bélgica $(61,73 \%)$. EGENOR tiene como accionista principal a Duke (99,9\%) de Estados Unidos.

Las tres empresas privadas tienen una mixtura diferenciada entre generación de energía por fuente hídrico y térmico. En el Gráfico N.$^{\circ} 4$ se presenta la estructura de la capacidad instalada de producción de energía de las cuatro empresas.

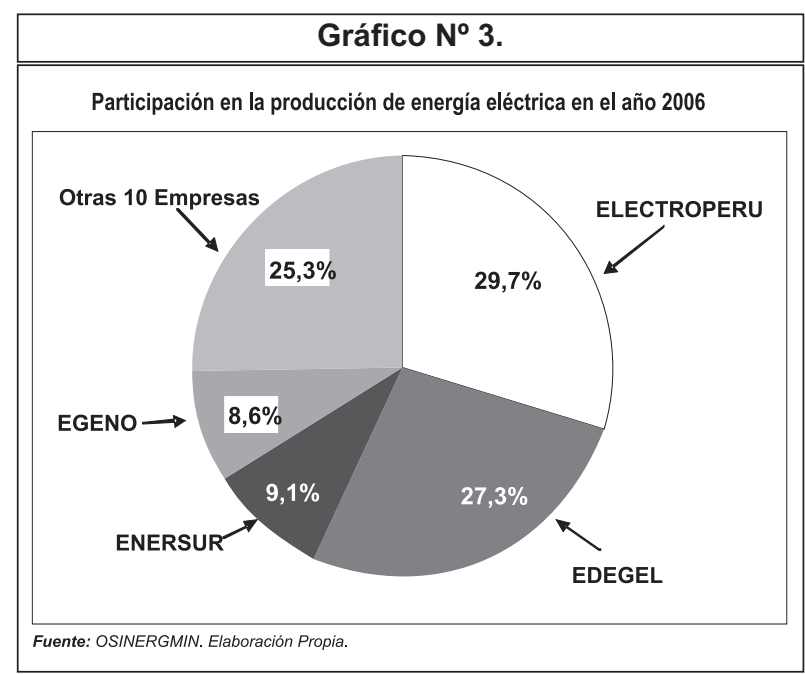

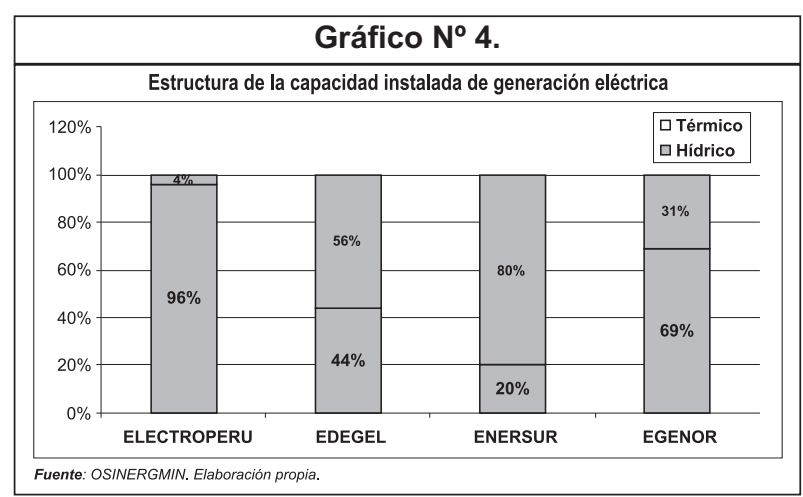

b. Resultados económicos y posición patrimonial

Según el Gráfico N. ${ }^{\circ} 5$, se observa que en los años 2006 y 2005 las cuatro empresas registraron utilidades. En el año 2006, ELECTROPERU obtuvo una utilidad neta de S/. 245,6 millones (33\% mayor que la del año 2005) y superior a la de las otras tres empresas. EDEGEL es la que tiene registra al cierre del año 2006, mayores saldos en activo y patrimonio, de S/. 4921,2 millones y S/. 2237 millones, respectivamente, y luego ELECTROPERU.

Para el año 2006, según el Gráfico N. ${ }^{\circ} 6$, la utilidad neta por acción ha sido en promedio del orden de S/. 0,13 por cada acción (similar al obtenido por ELECTROPERU). Los mayores rendimientos económicos unitarios corresponden a ENERSUR y ELECTROPERU.
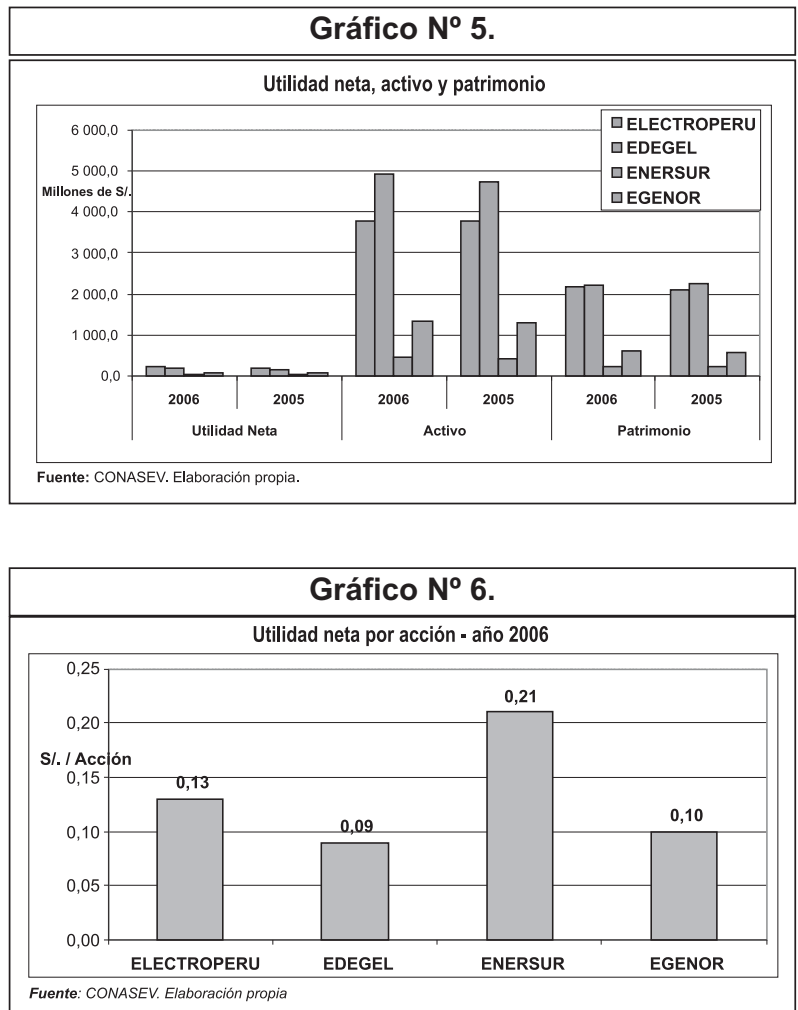


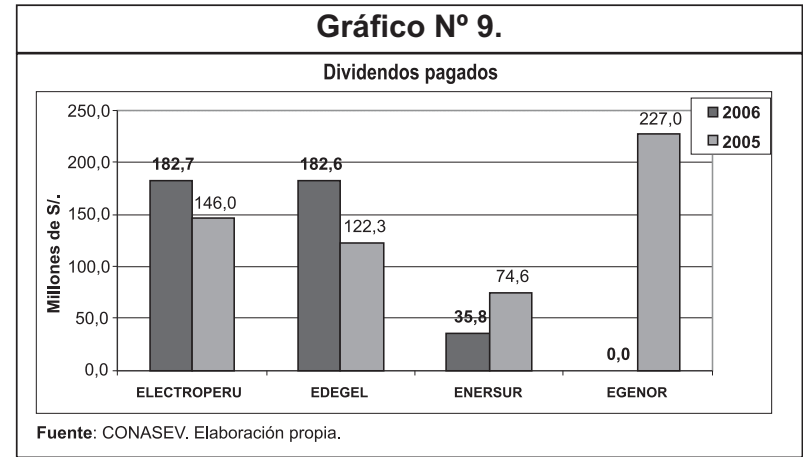

c. Dividendos pagados y valor de las acciones La política de dividendos es uniforme en ENERSUR, EDEGEL y EGENOR, la de distribuir el $100 \%$ de las utilidades de libre disposición provenientes del ejercicio económico del año, descontando los pagos de dividendos provisorios (anticipados). En ELECTROPERU, la política es distribuir dividendos como mínimo el $30 \%$ de la utilidad distribuible del ejercicio. En el Gráfico N. ${ }^{\circ}$ 7 se presentan los dividendos pagados por las cuatro empresas en los años 2006 y 2005.

El valor nominal de cada acción (capital social / número de acciones) de ELECTROPERU, ENERSUR y EGENOR es S/. 1, y de EDEGEL es S/. 0,90. En el Gráfico N. ${ }^{\circ}$ 8, se tiene el valor contable (Patrimonio / número de acciones), el cual depende de la política de dividendos y de nuevos aportes de capital que pudiesen registrar las empresas en cada periodo.

De otro lado, las acciones de ENERSUR y EDEGEL cotizan en la BVL; en cambio, las de EGENOR y ELECTROPERU solo están listadas. En EGENOR, el accionista mayoritario tiene 99,9\% de participación y en ELECTROPERU (100\% del FCR) se encuentra listada el $10 \%$ de su capital social. A fines del año 2006, la cotización de las acciones de ENERSUR y de EDEGEL eran de S/. 10,6 y S/. 1,40 por acción, que aumentan en $33 \%$ y $4 \%$ con respecto al cierre del ejercicio 2005, respectivamente. El precio de mercado de las acciones $(P)$ de ENERSUR supera ampliamente a su valor contable (VC), siendo la relación P/VC de 3,37 al cierre del año 2006.

\section{CONCLUSIONES}

El análisis de rentabilidad de una alternativa de inversión basada en el flujo de caja financiero (ingresos menos egresos operativos, financieros y de inversión), no resulta suficiente. Es conveniente ampliar el análisis incluyendo el efecto de la política de dividendos en la rentabilidad esperada por los accionistas.
La Junta General de Accionistas de la empresa debe aprobar la política de dividendos, en función a las posibilidades de la empresa para asumir nuevas obligaciones financieras que ayuden a su crecimiento mediante inversiones en activos y/o reestructuración de pasivos, y que contribuyan a generar valor para sus accionistas.

En el sector de generación eléctrica local, coexisten empresas estatales y privadas, en competencia y bajo la supervisión de un organismo estatal regulador para las operaciones productivas y comerciales de dichas empresas. Las cuatro empresas seleccionadas para el análisis, si bien aplican políticas de dividendos similares, vienen obteniendo diversos resultados económicos y financieros, según sus planes de desarrollo a mediano y largo plazos, lo cual resulta atractivo para sus accionistas, principalmente para aquellos que tienen el control mayoritario del capital social de las empresas.

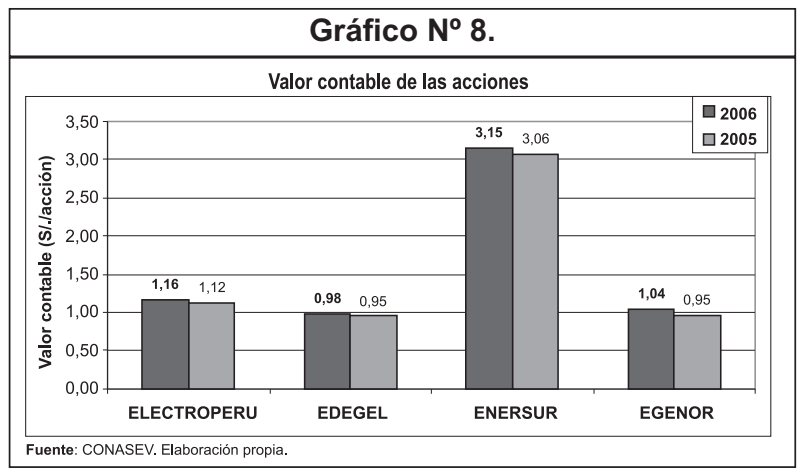

\section{REFERENCIAS BIBLIOGRÁFICAS}

Comisión Nacional Supervisora de Empresas y Valores - CONASEV (2007). Información financiera de las empresas listadas en la Bolsa de Valores de Lima. http://www.sbs.gob.pe/ portalsbs/tipotasa/indice.htm (visitado el 2007-09-21).

Fernández, P. (2002). Valoración de empresas. 1da ed. Ediciones Gestión 2000, España.

Moyer, R. Ch., McGuigan, J.R., Kretlow, W.J. (1998). Administración financiera contemporánea. $7 \mathrm{ma}$ ed. International Thomson Editores, México.

Organismo Supervisor de la Inversión en Energía y Minería - OSINERGMIN (2007). Anuario Estadístico 2006. Edición año 2007, Perú.

Superintendencia de Banca, Seguros y AFP - SBS (2007). Tasas de interés activas en el sistema bancario. http://www.sbs.gob.pe/portalsbs/tipotasa/ indice.htm (visitado el 2007-09-21). 